Abstract S33 Table 1 Main outcome measures at baseline and post intervention: change from baseline

\begin{tabular}{|c|c|c|c|c|c|}
\hline Outcome Measure & Group & $\begin{array}{l}\text { Baseline } \\
\text { mean(SD) } \\
\text { PAI, } n=27 \text { PR) }\end{array} \quad(n=23$ & $\begin{array}{l}\text { Post intervention } \\
\text { mean(SD) (PAI } \\
n=18, P R n=19)\end{array}$ & $\begin{array}{l}\text { Difference } \\
\text { change } \\
(95 \% \mathrm{Cl})\end{array}$ & Effect Size \\
\hline \multirow[t]{2}{*}{ Actigraph step count } & PAI & $\begin{array}{l}3305.65(1960.24) \\
N=17\end{array}$ & $\begin{array}{l}4768.21(2992.11) \\
N=14\end{array}$ & $\begin{array}{l}972.02 \quad(-1080.35 \\
\text { to } 3024.39)\end{array}$ & \multirow{2}{*}{0.42} \\
\hline & PR & $\begin{array}{l}3946.17(2263.11) \\
N=24\end{array}$ & $\begin{array}{l}3476.60(2307.87) \\
N=12\end{array}$ & $\begin{array}{l}4.26(-440.94 \text { to } \\
449.46)\end{array}$ & \\
\hline \multirow{2}{*}{$\begin{array}{l}\text { Total moderate- } \\
\text { vigourous PA }\end{array}$} & PAI & $\begin{array}{l}14.28(15.30) \\
N=17\end{array}$ & $\begin{array}{l}24.49(26.01) \\
N=14\end{array}$ & $\begin{array}{l}6.65 \\
23.68)\end{array}(-10.37$ to & \multirow{2}{*}{0.30} \\
\hline & PR & $\begin{array}{l}14.64(15.33) \\
N=24\end{array}$ & $\begin{array}{l}12.80(20.05) \\
N=12\end{array}$ & $0.86(-3.19$ to 4.90$)$ & \\
\hline \multirow{2}{*}{$\begin{array}{l}\text { Pedometer step } \\
\text { count }\end{array}$} & PAI & $\begin{array}{l}3044.43(1871.09) \\
\mathrm{N}=22\end{array}$ & $\begin{array}{l}5570.75(3486.70) \\
N=16\end{array}$ & $\begin{array}{l}2310.31 \text { (384.20 to } \\
4236.42)\end{array}$ & \multirow{2}{*}{0.77} \\
\hline & PR & $\begin{array}{l}3387.20(1942.80) \\
N=21\end{array}$ & $\begin{array}{l}3917.54(2194.95) \\
N=13\end{array}$ & $\begin{array}{l}146.91(-823.42 \text { to } \\
1117.23)\end{array}$ & \\
\hline \multirow[t]{2}{*}{$\begin{array}{l}\text { IPAQ Total PA level } \\
\text { (MET/mins/week) }\end{array}$} & PAI & $1464.07(1553.34)$ & $\begin{array}{l}2427.75(1559.72) \\
N=18\end{array}$ & $\begin{array}{l}907.50(-221.57 \text { to } \\
2036.57)\end{array}$ & \multirow{2}{*}{0.14} \\
\hline & PR & $1734.03(1692.63)$ & $\begin{array}{l}2229.86(2189.92) \\
N=18\left(\sum n=1\right)\end{array}$ & $\begin{array}{l}547.52(-827.74 \text { to } \\
1922.77)\end{array}$ & \\
\hline \multirow{2}{*}{$\begin{array}{l}\text { CAT }\left(0-4 O_{i} \text { a }\right. \\
\text { higher } \\
\text { indicates a higher } \\
\text { severity) }\end{array}$} & PAI & $23.83(6.86)$ & $\begin{array}{l}22.47(7.05) \\
n=17(* n=1)\end{array}$ & $0.65(-3.29$ to 4.58$)$ & \multirow{2}{*}{0.15} \\
\hline & PR & $19.41(8.01)$ & $16.58(5.26)$ & $\begin{array}{lll}-0.42 & (-3.51 & \text { to } \\
2.67) & & \\
\end{array}$ & \\
\hline \multirow{2}{*}{$\begin{array}{l}\text { ISWT Distance (M) } \\
\text { (O-1020m; a higher } \\
\text { score indicates a } \\
\text { higher exercise } \\
\text { capacity) }\end{array}$} & PAI & $253.04(118.84)$ & $\begin{array}{l}288.13(107.03) \\
n=16\left(\alpha n=1, \infty_{n=1}\right)\end{array}$ & $\begin{array}{l}-11.88 \\
36.31)\end{array} \quad(-60.06$ to & \multirow[t]{2}{*}{-0.05} \\
\hline & PR & $254.81(139.80)$ & $\begin{array}{l}267.65(144.50) \\
n=17\left(\sum n=1,{ }^{\circ} n=1\right)\end{array}$ & $\begin{array}{lll}-7.65 & (-43.57 & \text { to } \\
28.28) & & \end{array}$ & \\
\hline
\end{tabular}

\section{S34 EFFECT OF 8 AND 12 WEEKS' ONCE-DAILY TIOTROPIUM AND OLODATEROL, ALONE AND COMBINED WITH EXERCISE TRAINING, ON EXERCISE ENDURANCE DURING WALKING IN PATIENTS WITH COPD}

${ }^{1} \mathrm{~T}$ Troosters, ${ }^{2} \mathrm{~J}$ Bourbeau, ${ }^{3} \mathrm{~F}$ Maltais, ${ }^{4} \mathrm{~N}$ Leidy, ${ }^{5} \mathrm{D}$ Erzen, ${ }^{6} \mathrm{D}$ De Sousa, ${ }^{7} \mathrm{~L}$ Korducki, ${ }^{8} \mathrm{KL}$ Lavoie, ${ }^{9} \mathrm{~W}$ Janssens, ${ }^{6} \mathrm{~A}$ Hamilton. ${ }^{1} \mathrm{KU}$ Leuven, Department of Rehabilitation Sciences and University Hospital Leuven, Pulmonary Rehabilitation and Respiratory Division, Leuven, Belgium; ${ }^{2}$ McGill University Health Centre, Montreal, Canada; ${ }^{3}$ Centre de Recherche, Institut Universitaire de Cardiologie et de Pneumologie de Québec, Université Laval, Laval, Canada; ${ }^{4}$ Evidera, Bethesda, USA; ${ }^{5}$ Boehringer Ingelheim Pharma GmbH and Co. KG, Biberach an der Riss, Germany; ${ }^{6}$ Boehringer Ingelheim (Canada) Ltd, Burlington, Canada; ${ }^{7}$ Boehringer Ingelheim Pharmaceuticals Inc, Ridgefield, USA; ${ }^{8}$ Montreal Behavioural Medicine Centre, Research Centre, Hopital du Sacré-Coeur de Montreal, Montreal, Canada; ${ }^{9}$ University Hospital Gasthuisberg, Respiratory Division, Leuven, Belgium

\subsection{6/thoraxjnl-2016-209333.40}

Rationale Physical deconditioning is common in patients with chronic obstructive pulmonary disease (COPD), limiting exercise tolerance. PHYSACTO ${ }^{\circledast}$ (NCT02085161) tested the effects of long-acting bronchodilators alone or combined with exercise training (ExT) on exercise endurance time (EET) in patients with COPD. All patients took part in a standardised physical activity self-management behaviour-modification (BM) programme.

Methods A 12-week, randomised, partially double-blind, placebo-controlled, parallel-group trial at 34 sites in Australia, New Zealand, USA, Canada and Europe. Interventions (all with 12week BM) were: $\mathrm{BM}+$ placebo; $\mathrm{BM}+$ tiotropium (T) $5 \mu \mathrm{g}$; BM $+\mathrm{T}+$ olodaterol $(\mathrm{T} / \mathrm{O}) 5 / 5 \mu \mathrm{g} ; \mathrm{BM}+\mathrm{T} / \mathrm{O} 5 / 5 \mu \mathrm{g}$ with 8 weeks' ExT $(\mathrm{T} / \mathrm{O} 5 / 5 \mu \mathrm{g}+\mathrm{ExT})$. EET (log transformed) during an endurance shuttle-walk test (ESWT) to symptom limitation was assessed after 8 weeks (primary end point) and 12 weeks.
Results 303 patients (200 men) were randomised and treated (full analysis set $\mathrm{n}=274$ ). Mean post-bronchodilator forced expiratory volume in 1 second was $1.59 \mathrm{~L}$ (57\% predicted). EET was significantly increased in patients receiving $\mathrm{BM}+\mathrm{T} / \mathrm{O} 5 / 5 \mu \mathrm{g}$

Abstract S34 Table 1

\begin{tabular}{|c|c|c|c|}
\hline \multirow[t]{2}{*}{ Intervention arm } & \multicolumn{3}{|c|}{$\begin{array}{l}\text { EET, treatment comparison } \\
\text { vs } \mathrm{BM}+\text { placebo }\end{array}$} \\
\hline & $\begin{array}{l}\text { EET, adjusted mean } \pm \\
S E \text {, seconds }\end{array}$ & $\begin{array}{l}\text { Adjusted mean difference } \pm \\
\text { SE, seconds }\end{array}$ & $95 \% \mathrm{Cl}$ \\
\hline Week 8 & $355.73 \pm 24.787$ & $1.458 \pm 0.147^{c}$ & 1.196, \\
\hline$B M+T / O+E x T$ & $315.32 \pm 21.671$ & $1.292 \pm 0.129^{a}$ & 1.777 \\
\hline$(n=70)$ & $254.18 \pm 18.099$ & $1.041 \pm 0.106$ & 1.061, \\
\hline$B M+T / O$ & $244.07 \pm 17.666$ & & 1.573 \\
\hline$(n=72)$ & & & 0.853 \\
\hline$B M+T(n=67)$ & & & 1.272 \\
\hline \multicolumn{4}{|l|}{ BM + placebo } \\
\hline \multicolumn{4}{|l|}{$(n=65)$} \\
\hline Week 12 & $324.21 \pm 24.095$ & $1.333 \pm 0.142^{b}$ & 1.080 \\
\hline$B M+T / O+E x T$ & $302.61 \pm 21.691$ & $1.244 \pm 0.131^{\mathrm{a}}$ & 1.645 \\
\hline$(n=66)$ & $255.67 \pm 19.292$ & $1.051 \pm 0.113$ & 1.011 \\
\hline $\mathrm{BM}+\mathrm{T} / \mathrm{O}$ & $243.30 \pm 18.680$ & & 1.530 \\
\hline$(n=71)$ & & & 0.850 \\
\hline$B M+T(n=64)$ & & & 1.299 \\
\hline \multicolumn{4}{|l|}{ BM + placebo } \\
\hline$(n=62)$ & & & \\
\hline
\end{tabular}


compared to placebo and was increased to a greater extent in patients receiving $\mathrm{BM}+\mathrm{T} / \mathrm{O} 5 / 5 \mu \mathrm{g}+$ ExT compared to placebo at 8 weeks. EET was not significantly increased in patients receiving $\mathrm{BM}+\mathrm{T} 5 \mu \mathrm{g}$ compared to placebo (Table). Similar increases in EET were demonstrated at 12 weeks (Table). 13 patients reached test termination criteria (20 minutes) without symptom limitation (placebo, $\mathrm{n}=0 ; \mathrm{T}, \mathrm{n}=3 ; \mathrm{T} / \mathrm{O}, \mathrm{n}=2 ; \mathrm{T} / \mathrm{O}+\operatorname{ExT}, \mathrm{n}=8$ ) at 8 weeks. No safety concerns were identified.

Conclusions When added to $\mathrm{BM}$, the bronchodilator combination $\mathrm{T} / \mathrm{O} 5 / 5 \mu \mathrm{g}$, used alone and combined with ExT, improved EET during ESWT compared to placebo in moderate to severe COPD.

Funding Boehringer Ingelheim.

Please refer to page A270 for declarations of interest in relation to abstract S34.

\section{S35 EFFICACY AND SAFETY OF THE DIRECT SWITCH FROM VARIOUS PREVIOUS TREATMENTS TO GLYCOPYRRONIUM OR INDACATEROL/ GLYCOPYRRONIUM IN PATIENTS WITH MODERATE COPD: THE CRYSTAL STUDY}

${ }^{1} \mathrm{C}$ Vogelmeier, ${ }^{2} \mathrm{M}$ Aalamian-Mattheis, ${ }^{1} \mathrm{~T}$ Greulich, ${ }^{3} \mathrm{~J} \mathrm{M}$ Marin, ${ }^{4} \mathrm{~W}$ Castellani, ${ }^{5} \mathrm{~T}$ Similowski, ${ }^{6} \mathrm{~V}$ Ninane, ${ }^{7} \mathrm{M}$ Gaga, ${ }^{8} \mathrm{~S}$ Lane, ${ }^{9} \mathrm{X}$ Nunez, ${ }^{2} \mathrm{~F}$ Patalano, ${ }^{2} \mathrm{~A}$ Clemens, ${ }^{2} \mathrm{~K}$ Kostikas. 'University Medical Centre Giessen and Marburg, Marburg, Germany; ${ }^{2}$ Novartis Pharma AG, Basel, Switzerland; ${ }^{3}$ Respiratory Medicine, Hospital Universitario Miguel Servet, Zaragoza, Spain; ${ }^{4}$ Department of Respiratory Physiopathology, Palagi Hospital, Florence, Italy; ${ }^{5}$ Respiratory Medicine and Intensive Care, Pitié - Salpêtrière Hospital, Paris, France; ${ }^{6} \mathrm{CHU}$ Saint-Pierre - Service de Pneumologie, Brussells, Belgium; ${ }^{7}$ Athens Chest Hospital "Sotiria", Athens, Greece; ${ }^{8}$ Adelaide and Meath Hospital, Dublin, Ireland; ${ }^{9}$ TFS Develop, Barcelona, Spain

\subsection{6/thoraxjnl-2016-209333.41}

Introduction and objectives In contrast to clinical trials, changes to new therapies in clinical practice occur without any washout period. The CRYSTAL study was designed to mimic clinical practice. Patients with symptomatic, non-frequently exacerbating, moderate COPD treated with various drugs were directly switched to glycopyrronium $50 \mu \mathrm{g}$ (GLY) or indacaterol/glycopyrronium 110/50 $\mu \mathrm{g}$ (IND/GLY). Lung function and symptoms were evaluated.

Methods CRYSTAL was a prospective, multicentre, 12-week, randomised, pragmatic, open-label trial. Patients were recruited into 4 Groups according to previous medication and symptoms (mMRC) and randomised to a direct switch to GLY or IND/GLY vs. continuation of baseline therapy $(3: 1)$. Co-primary objectives were superiority of GLY vs. previous SABA and/or SAMA, noninferiority of GLY vs. previous LABA or LAMA, and superiority of IND/GLY vs. LABA, LAMA and LABA+ICS regarding trough FEV1 and transition dyspnoea index (TDI) at Week 12. Due to slow recruitment, Groups A and B were prematurely discontinued at the time of completion of Groups C and D.

Results Of the 4,389 patients randomised, 2,159 patients received IND/GLY (C2: $\mathrm{n}=811$; D2: $\mathrm{n}=811$ ) or continued their previous treatment (LABA + ICS C1: $\mathrm{n}=269$; LABA or LAMA D1: $\mathrm{n}=268)$. IND/GLY provided superior improvement in trough FEV1 at Week 12 vs. LABA + ICS (treatment difference $(\Delta)=71 \mathrm{~mL}, \mathrm{p}<0.0001)$ and LABA or LAMA $(\Delta=101$ $\mathrm{mL}, \mathrm{p}<0.0001)$. IND/GLY also improved TDI vs. LABA + ICS $(\Delta=1.10$ units, $\mathrm{p}<0.0001)$ and vs. LABA or LAMA $(\Delta=1.26$ units, $\mathrm{p}<0.0001)$. Significantly more patients on IND/GLY reached the minimally clinically important difference (MCID) of $100 \mathrm{~mL}$ for trough FEV1 and 1 point for TDI vs. comparators (Table 1). In the Groups A and B that were underpowered due to sample size, GLY was superior to previous SABA and/or SAMA and was non-inferior to previous LABA or LAMA on trough FEV1 and TDI (Table 1). GLY and IND/GLY were well tolerated. Conclusions In the pragmatic CRYSTAL trial, IND/GLY demonstrated superior improvement in lung function (trough FEV1) and dyspnoea (TDI) after 12 weeks, in symptomatic patients with moderate COPD and a history of up to 1 exacerbation in the previous year, after direct switch from previous treatment with either LABA+ICS or with a LABA or LAMA.

Abstract S35 Table 1 CRYSTAL Study Results

\begin{tabular}{|c|c|c|c|c|c|c|c|c|}
\hline & $\begin{array}{l}A 1 \\
(n=122)\end{array}$ & $\begin{array}{l}A 2 \\
(n=369)\end{array}$ & $\begin{array}{l}\text { B1 } \\
(n=420)\end{array}$ & $\begin{array}{l}\text { B2 } \\
(n=1254)\end{array}$ & $\begin{array}{l}\text { C1 } \\
(n=269)\end{array}$ & $\begin{array}{l}\text { C2 } \\
(n=811)\end{array}$ & $\begin{array}{l}\text { D1 } \\
(n=268)\end{array}$ & $\begin{array}{l}\text { D2 } \\
(n=811)\end{array}$ \\
\hline Trough FEV F $_{1}(\mathrm{~L})$ & $\begin{array}{l}1.826 \\
{[1.780,1.873]}\end{array}$ & $\begin{array}{l}1.892 \\
{[1.865,1.919]}\end{array}$ & $\begin{array}{l}1.800 \\
{[1.777,1.824]}\end{array}$ & $\begin{array}{l}1.822 \\
{[1.808,1.835]}\end{array}$ & $\begin{array}{l}1.685 \\
{[1.654,1.715]}\end{array}$ & $\begin{array}{l}1.756 \\
{[1.738,1.774]}\end{array}$ & $\begin{array}{l}1.673 \\
{[1.646,1.699]}\end{array}$ & $\begin{array}{l}1.774 \\
{[1.759,1.790]}\end{array}$ \\
\hline Differences in trough $\mathrm{FEV}_{1}(\mathrm{~L})$ & $\begin{array}{l}0.065 \\
{[0.011,0.119]^{*}}\end{array}$ & & $\begin{array}{l}0.021 \\
{[-0.006,0.048]^{\#}}\end{array}$ & & $\begin{array}{l}0.071 \\
{[0.036,0.107]^{* *}}\end{array}$ & & $\begin{array}{l}0.101 \\
{[0.071,0.132]^{* *}}\end{array}$ & \\
\hline Patients with MCID in trough $\mathrm{FEV}_{1}(\geq 100 \mathrm{~mL})^{\dagger}$ & $\begin{array}{l}1.770 \\
(1.150,2.724)\end{array}$ & & $\begin{array}{l}1.401 \\
(1.092,1.798)\end{array}$ & & $\begin{array}{l}1.902 \\
(1.421,2.546)\end{array}$ & & $\begin{array}{l}2.526 \\
(1.863,3.424)\end{array}$ & \\
\hline TDI & $\begin{array}{l}0.51 \\
{[-0.01,1.03]}\end{array}$ & $\begin{array}{l}2.30 \\
{[2.00,2.60]}\end{array}$ & $\begin{array}{l}0.70 \\
{[0.42,0.98]}\end{array}$ & $\begin{array}{l}1.44 \\
{[1.27,1.60]}\end{array}$ & $\begin{array}{l}0.90 \\
{[0.47,1.23]}\end{array}$ & $\begin{array}{l}1.95 \\
{[1.72,2.18]}\end{array}$ & $\begin{array}{l}0.90 \\
{[0.51,1.22]}\end{array}$ & $\begin{array}{l}2.12 \\
{[1.91,2.33]}\end{array}$ \\
\hline Differences in TDI & $\begin{array}{l}1.79 \\
{[1.19,2.39]^{* *}}\end{array}$ & & $\begin{array}{l}0.74 \\
{[0.41,1.06]^{\#}}\end{array}$ & & $\begin{array}{l}1.10 \\
{[0.652,1.55]^{* *}}\end{array}$ & & $\begin{array}{l}1.26 \\
{[0.848,1.67]^{* *}}\end{array}$ & \\
\hline Patients with MCID in TDI ( $\geq 1$ point $)^{\dagger}$ & $\begin{array}{l}4.58 \\
{[2.86,7.34]}\end{array}$ & & $\begin{array}{l}2.57 \\
{[2.00,3.29]}\end{array}$ & & $\begin{array}{l}2.609 \\
{[1.94,3.50]}\end{array}$ & & $\begin{array}{l}2.853 \\
{[2.13,3.82]}\end{array}$ & \\
\hline
\end{tabular}

All data are LSM Data with [95\% Cl], unless otherwise stated

†Odds ratios are displayed with $[95 \% \mathrm{Cl}]$

Linear mixed model. All primary efficacy analysis are superiority analysis except B1-B2 which are non-inferiority analysis.

Group B: non-inferiority testing $\left(\Delta:-40 \mathrm{~mL}\right.$ for trough $\mathrm{FEV}_{1}$ and -0.6 points for TDI)

${ }^{*} \mathrm{p}<0.05 ;{ }^{* *} \mathrm{p}<0.0001 ;{ }^{*} \mathrm{p}<0.0001$ (non-inferiority)

A1 (any SABA and/or SAMA), A2 (GLY), B1 (any LAMA or LABA and mMRC $=1), B 2(G L Y$ and $m M R C=1)$

C1 (any LABA and ICS), C2 (IND/GLY), D1 (any LAMA or LABA and mMRC > 1), D2 (IND/GLY and mMRC > 1)

$\mathrm{Cl}$ : confidence intervals; LSM: least square means; OR: odds ratio; MCID, minimal clinically important difference 